\title{
Cosmological Daemon
}

\author{
I.Ya. Aref'eva \\ Steklov Mathematical Institute, Russian Academy of Sciences, \\ Gubkina str. 8, 119991, Moscow, Russia \\ arefeva@mi.ras.ru
}

\section{I.V. Volovich \\ Steklov Mathematical Institute, Russian Academy of Sciences, Gubkina str. 8, 119991, Moscow, Russia \\ volovich@mi.ras.ru}

\begin{abstract}
Classical versions of the Big Bang cosmological models of the universe contain a singularity at the start of time, hence the time variable in the field equations should run over a half-line. Nonlocal string field theory equations with infinite number of derivatives are considered and an important difference between nonlocal operators on the whole real line and on a half-line is pointed out. We use the heat equation method and show that on the half-line in addition to the usual initial data a new arbitrary function (external source) occurs that we call the daemon function. The daemon function governs the evolution of the universe similar to Maxwell's demon in thermodynamics. The universe and multiverse are open systems interacting with the daemon environment. In the simplest case the nonlocal scalar field reduces to the usual local scalar field coupled with an external source which is discussed in the stochastic approach to inflation. The daemon source can help to get the chaotic inflation scenario with a small scalar field.
\end{abstract}

Keywords: Cosmology of Theories beyond the SM, Nonlocal Cosmology. 


\section{Contents}

1. Introduction 2

2. Nonlocal String Field and p-adic String Actions 田

3. Nonlocal operator $e^{\partial_{t}^{2}}$ and heat equation 6

4. Dirichlet's Daemon and Neumann's Daemon 6

5. Laplace Daemon 8

6. Solutions to Linearized Nonlocal Equation on the Half-line 9

6.1 Dirichlet's daemon without source 9

6.2 Dirichlet's daemon with source 11

6.3 Neumann's daemon without source 11

7. Nonlinear Tachyon Equation on the Whole Line 12

8. Nonlinear Nonlocal Equations on the Half-line 13

8.1 Dirichlet's case

8.2 Neumann's case 14

9. Nonlocal Equation on the Half-line in Friedmann Cosmology 15

9.1 Dirichlet's case 16

9.2 Neumann's case 16

9.3 Solutions to Linearized Nonlocal Equation on the Half-line, $H_{0} \neq 0$

10. Inflation and External Sources 17

11. Conclusion $\quad 20$

12. Acknowledgements 20

丹. Laplace transformation and definition of $F\left(\partial_{t}\right)$

B. Proof of proposition 1 . 


\section{Introduction}

There are many considerations of various types of boundary conditions in classical and quantum cosmology [1, 2, 3, 4, Classical versions of the Big Bang cosmological models of the universe contain a singularity at the beginning of time, hence the time variable in the field equations should run over a half-line ${ }^{1}$.

In this paper we consider nonlocal string field theory equations with infinite number of derivatives and point out an important difference between nonlocal operators on the whole real line and on a half-line. We use the heat equation method and show that on the half-line in addition to the usual initial data a new arbitrary function (external source) occurs that we call the daemon function ${ }^{2}$. The daemon function governs the evolution of the universe similar to Maxwell's demon in statistical physics which was created to show that the second law of thermodynamics has only a statistical certainty. In the present approach the universe and even the Multiverse are open systems interacting with the daemon environment.

In the simplest case the nonlocal scalar field reduces to the usual local scalar field coupled with an external source. The daemon source can help to get the chaotic inflation scenario with a small scalar field.

We shall consider the following string field theory effective action [6] describing the nonlocal scalar field coupled with gravity:

$$
S=\int d^{4} x \sqrt{-g}\left\{\frac{m_{p}^{2}}{2} R+\Phi F(\square) \Phi-V(\Phi)\right\},
$$

where $F(\square)$ is a function of the D'Alembert operator, $\square=\frac{1}{\sqrt{-g}} \partial_{\mu} \sqrt{-g} g^{\mu \nu} \partial_{\nu}, g_{\mu \nu}$ is the metric, $R$ is the scalar curvature, $m_{p}$ is a rescaled Planck mass, $\Phi$ is a scalar field and $V(\Phi)$ is a potential. Cosmological applications of various forms of such action have been considered in [6]-49].

Nonlocal operators that appear in string field theory [50] and in $p$-adic strings [51, 52, 53] $]^{3}$ usually include the exponential function of the D'Alembert operator, $e^{-\square}$.

There are two important properties of the function $F(z)$ to be discussed. First, if the equation $F(z)=0$ has a number of roots $\left\{z=m_{n}^{2}\right\}$ then our theory with the nonlocal field $\Phi$ is equivalent to the theory with the corresponding number of local fields $\phi_{n}$ 12, 13, 16, 17, 25, 32.

\footnotetext{
${ }^{1}$ Rigorous mathematical definition of singularities in General Relativity requires some boundary constructions, points at infinity and so on [5]. Here we restrict ourself with a simple approach by considering the time variable $t$ running over the half-line $[0, \infty)$ with regular boundary conditions at $t=0$.

${ }^{2}$ According to Plato, daemons are good or benevolent "supernatural beings between mortals and gods, such as inferior divinities", and differ from the Judeo-Christian usage of demon that is a malignant spirit. Socrates' daimon is analogous to the guardian angel.

${ }^{3} p$-adic cosmology is considered in [55].
} 
Second, and it is the subject of this paper, if the function $F(\square)$ includes the

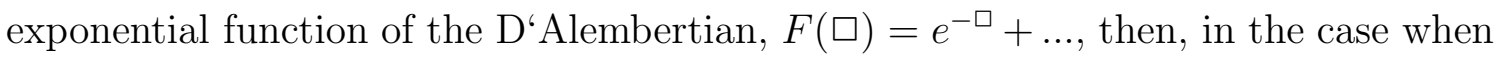
we work on the half-line $t>0$, an additional freedom in the boundary conditions at the point $t=0$ occurs, besides the usual initial data. In the homogenous case we have to define the action of the operator $e^{\partial_{t}^{2}}$ on the function $\varphi(t)$. To this end, one can use an auxiliary function of two variables $\Psi(\tau, t)=e^{\tau \partial_{t}^{2}} \varphi(t)$ satisfying the heat equation

$$
\left(\partial_{\tau}-\partial_{t}^{2}\right) \Psi(t, \tau)=0,
$$

with the initial condition

$$
\left.\Psi(t, \tau)\right|_{\tau=0}=\varphi(t) .
$$

Then the action of $e^{\partial_{t}^{2}}$ on the function $\varphi(t)$ is defined by the solution to (1.2) with the initial data (1.3) as follows

$$
e^{\partial_{t}^{2}} \varphi(t)=\left.\Psi(t, \tau)\right|_{\tau=1} .
$$

This prescription works if the time variable $t$ runs over the whole real line. The heat equation (diffusion equation) on the whole line is an efficient method to study the rolling tachyon solutions in flat space [68, 69, 70, 56, 57, 30] as well as in the FRW cosmology [21, 32, 33, 41]. It is important to note that on the half-line $t>0$ we have to add to the initial data (11.3) also the boundary condition at $t=0$ with some function $\mu$.

We consider the heat equation (1.2) on the half-line $t>0$ and $\tau>0$ with the initial data (1.3) and the following boundary condition at $t=0$ :

$$
\Psi(0, \tau)=\mu(\tau) .
$$

This is the mixed initial-boundary value Dirichlet's problem for the heat equation on the half-line. The function $\mu(\tau)$ will be called the Dirichlet daemon function. It describes the Dirichlet boundary conditions at $t=0$. On the half-line $t>0$ we define the action of the operator $e^{\partial_{t}^{2}}$ on the function $\varphi(t)$ as $e^{\partial_{t}^{2}} \varphi(t)=\left.\Psi(t, \tau)\right|_{\tau=1}$, where $\Psi(t, \tau)$ is the solution of the initial-boundary value problem (1.2), (11.3), (11.5). Now the result $e^{\partial_{t}^{2}} \varphi(t)$ depends on the function $\mu$, see Sect.3.

For the nonlocal action (1.1) in addition to the local fields $\phi_{n}$ the new arbitrary function $\mu$ appears which is an external source. The simplest version of the local action with an external source has the form

$$
S=\int d^{4} x \sqrt{-g}\left\{\frac{M_{P}^{2}}{2} R+\frac{1}{2} \phi \square \phi-U(\phi)+J \phi\right\},
$$

where $J=J(x)$ is the external source and $U(\phi)$ is the potential. We shall discuss the inflation scenario for this action in the conclusion. 
Note that if the source $J(x)$ is a white noise then the equations of motion for the local action (1.6) are reduced to the Langevin equations in the stochastic approach to inflation introduced by Starobinsky [54].

In mathematical literature equations with infinite number of derivatives have been considered in [56]-61] (see also [62, 63, 64, 65]), but, as we are aware of, the appearance of the additional boundary function $\mu$ on the half-line for the exponential operator was not discussed. Integral equations at the half-line are investigated in [66] (and ref's therein).

The operator $e^{\partial_{t}^{2}}$ on the half axis by using the Laplace transform has been considered in the stimulating paper by Barnaby and Kamran [22]. Note that the Mellin transform has been also used in the investigation of the quantum Riemann function $\zeta(\square)$ [17]. In the present paper we clarify the relation between these two approaches: the Laplace transform and the heat equation. We show in Sect.5 that the Laplace transform method is a special case of the more general heat equation method and it does not include an arbitrary function $\mu(\tau)$ at the boundary $t=0$. The Laplace transform method corresponds to a special choice of the daemon function $\mu(\tau)$ that is uniquely defined by the function $\varphi(t)$.

The paper is organized as follows. In Sect.2 we remind the form of nonlocal string field theory and p-adic string actions. In Sect.3 we present the definition of the operator $e^{-\square}$ via the heat (diffusion) equation and stress the difference between the diffusion equation on the whole real line and on the half-line. In Sect.4 we show that on the half-line in addition to the usual initial data a new arbitrary function (external source) occurs that we call the daemon function. Depending of the type of the boundary conditions, the Dirichlet or Neumann, we deal with different definitions of the operator $e^{\partial_{t}^{2}}$. In Sect.5 we show that interpretation of the operator $e^{\partial_{t}^{2}}$ as a symbol via Laplace transform corresponds to the Dirichlet boundary condition with a special source. In Sect.6 we present solutions to a linearized nonlocal equation corresponding to the Dirichlet and Neumann boundary conditions. In Sects.7 and 8 we describe approximate solutions to nonlinear equations. In Sect. 9 we give a generalization of the previous results to the case of the FRW metric. In Sect.10 we sketch possible applications of the external daemon source for inflation scenario, namely we discuss the chaotic and stochastic approaches to inflation in the presence of an external source.

\section{Nonlocal String Field and p-adic String Actions}

The level truncated string field action for the tachyon leaving on the 3-brane and interacting in a minimal way with gravity has the form [6]

$$
S=\int d^{4} x \sqrt{-g}\left\{M_{p}^{2} \frac{R}{2}+\frac{1}{\alpha^{\prime 2} g_{o}^{2}}\left(\frac{\alpha^{\prime}}{2} \Phi\left(\square+\frac{1}{\alpha^{\prime}}\right) e^{-\kappa \alpha^{\prime} \square} \Phi-V(\Phi)\right)\right\} .
$$


Here $\alpha^{\prime}$ is a square of the characteristic length of the string and $1 / \alpha^{\prime}$ defines the mass of tachyon, $\Phi$ is a dimensionless tachyon field, $V(\Phi)$ is the tachyon potential, $V(\Phi)=\frac{1}{3} \Phi^{3}$ for the bosonic string, $V(\Phi)=\frac{1}{4} \Phi^{4}$ for the fermionic string, $g_{0}^{2}$ is the dimensionless open string coupling constant, $\kappa$ is a numerical parameter that is fixed by the string field theory, $\kappa=2 \ln (4 / 3 \sqrt{3})$.

The $p$-adic open string tachyon action has the form

$$
S=\int d^{4} x \sqrt{-g}\left\{M_{p}^{2} \frac{R}{2}-\frac{1}{\alpha^{\prime 2} g_{p}^{2}}\left(\Phi e^{-\kappa_{p} \alpha^{\prime} \square} \Phi-V(\Phi)\right)\right\} .
$$

Here we also assume that the characteristic scale of the theory is $\alpha^{\prime}, g_{p}$ is a coupling constant, $V(\Phi)=\frac{1}{p+1} \Phi^{p+1}$.

Using dimensionless coordinates, $x / \sqrt{\alpha^{\prime}}$ and performing recsaling one rewrites these actions in the form

$$
S=\int d^{4} x \sqrt{-g}\left\{m_{p}^{2} \frac{R}{2}+\frac{1}{\gamma}\left(\frac{1}{2} \Phi\left(\xi^{2} \square+1\right) e^{-\square} \Phi-V(\Phi)\right)\right\} .
$$

Here $m_{p}^{2}=\frac{M_{p}^{2}}{M_{s}^{2}}, \gamma=g_{o}^{2}$ for action (2.1) and $\gamma=-g_{p}^{2}<0$ in the p-adic inflation models [18, 19]. Note that in a naive local approximation $\left(e^{-\square} \approx 1-\square\right)$ the case $\xi=0, V=0$ with $\gamma<0$ corresponds to a massive field and with $\gamma>0$ corresponds to a ghost field with a negative mass term.

Therefore, string field theory (SFT) and $p$-adic string lead to (1.1) with the following form of $F(\square)$, [6]

$$
F(\square)=\left(\xi^{2} \square+1\right) e^{-\alpha \square}-\beta .
$$

As it has been mentioned in Introduction there are two possibilities to understand $e^{-\frac{1}{4} \square}$ as an operator on the whole real line and only on the semi-line.

It has been shown, [13, 16, 17] that the action (11.1) with the nonlocal field $\Phi$ on the whole axis is equivalent, modulo total derivatives, to the following action with an infinite number of local fields $\psi_{n}$

$$
S=\int d^{4} x \sqrt{-g}\left\{\frac{M_{P}^{2}}{2} R+\frac{1}{2} \sum_{n}\left[\eta_{n} \psi_{n} e^{f(\square)}\left(\square-\alpha_{n}^{2}\right) \psi_{n}+\text { c.c. }\right]-U\left(\left\{\psi_{n}\right\}\right)\right],
$$

where $\eta_{n}, \alpha_{n}$ are constants and $f$ is a function. This is a generalization of the similar flat result [67]. To get this representation the following Weierstrass product for the function $F(z)$ has been used: $F(z)=e^{f(z)} \prod_{n}\left(z-\alpha_{n}^{2}\right)$ (more general Weierstrass representations are discussed in [16, [17]).

In particular, for $\xi=0$

$$
F(\square)=e^{-\alpha \square}-\beta,
$$

where $\alpha, \beta$ are constants, there is the following Weierstrass representation, see [13]

$$
F(\square)=e^{-\alpha \square}-\beta=-\beta^{1 / 2} e^{-\alpha \square / 2}(\alpha \square+\log \beta) \prod_{j=1}^{\infty}\left(1+\frac{(\alpha \square+\log \beta)^{2}}{4 \pi^{2} j^{2}}\right) .
$$




\section{Nonlocal operator $e^{\partial_{t}^{2}}$ and heat equation}

The D'Alembert operator in Minkowski space has the form

$$
\square=-\partial_{t}^{2}+\partial_{x_{i}} \partial_{x_{i}}
$$

In the homogenous case we have to give a mathematical meaning to the operator $e^{\partial_{t}^{2}}$. To this end many authors have used the Fock auxiliary parameter method [71, 72]. This method is known also as the heat equation method, or the diffusion equation method. This method uses an auxiliary function of two variables $\Psi(\tau, t)=e^{\tau \partial_{t}^{2}} \varphi(t)$ satisfying the heat (diffusion) equation [56, 57, 68, 21, 36, 39, 41]

$$
\left(\partial_{\tau}-\partial_{t}^{2}\right) \Psi(t, \tau)=0
$$

and the initial condition

$$
\left.\Psi(t, \tau)\right|_{\tau=0}=\varphi(t) .
$$

Note that originally $t$ is temporal variable but here in the heat equation the variable $t$ plays the role of the spatial variable and $\tau$ is "time". Then the action of $e^{\partial_{t}^{2}}$ on a function $\varphi(t)$ is defined by means of the solution to (3.2) with the boundary condition (3.3) as follows

$$
\left.e^{\partial_{t}^{2}} \varphi(t) \equiv \Psi(t, \tau)\right|_{\tau=1}
$$

Now it is important to distinguish two different cases:

- i) whole real line $-\infty<t<\infty$,

- ii) half-line $0<t<\infty$.

In quantum field theory in Minkowski space-time the time variable $t$ runs from $-\infty$ to $\infty$. If we are interested in application of SFT equations, or p-adic string equations to cosmology [6, 14, 18] it is important to stress that there is a reason to restrict the time variable to the half axis only, $t>0$, since in the Friedman cosmology there is a cosmological singularity at $t=0[5]^{4}$.

\section{Dirichlet's Daemon and Neumann's Daemon}

In the case of the whole real axis under suitable assumptions there is a unique solution of the problem (3.2), (3.3). However, in the case of the half-line one has to add an extra boundary condition at $t=0$, see for example [74]. Therefore, we shall consider in this note the following mixed initial-boundary value problem:

$$
\left\{\begin{array}{l}
\frac{\partial}{\partial \tau} \Psi_{D}(t, \tau)=\frac{\partial^{2}}{\partial t^{2}} \Psi_{D}(t, \tau), \quad t>0, \quad \tau>0 \\
\Psi_{D}(t, 0)=\varphi(t) \\
\Psi_{D}(0, \tau)=\mu(\tau)
\end{array}\right.
$$

\footnotetext{
${ }^{4}$ Bouncing solutions can be extended to the whole real line 8
} 
This is the Dirichlet's problem for the heat equation on the half-line. The function $\mu(\tau)$ will be called the Dirichlet daemon function. It describes the Dirichlet boundary conditions at $t=0$. Our goal is to evaluate the contribution of the function $\mu(\tau)$ in the definition of the operator $e^{\partial_{t}^{2}}, t>0$ to cosmological applications. In the heat equation the physical meaning of the boundary function $\mu$ is that it describes of how the temperature at the boundary depends on time. In our case the role of time plays an auxiliary parameter $\tau$.

We define the action of the operator $e^{\tau \partial_{t}^{2}}$ on the half axis as follows

$$
e^{\tau \partial_{t}^{2}} \varphi(t)=\Psi_{D}(t, \tau), \quad t>0, \tau>0,
$$

where $\Psi_{D}(t, \tau)$ is a solution to (4.1). To solve the Dirichlet problem for the heat equation on the half-line one uses the odd extension of $\varphi$ to the whole real line. It is known [74] that the solution of (4.1) is given by

$$
\begin{aligned}
\Psi_{D}(t, \tau) & =\frac{1}{\sqrt{4 \pi \tau}} \int_{0}^{\infty} \varphi\left(t^{\prime}\right)\left[e^{-\frac{\left(t-t^{\prime}\right)^{2}}{4 \tau}}-e^{-\frac{\left(t+t^{\prime}\right)^{2}}{4 \tau}}\right] d t^{\prime} \\
& +\frac{t}{\sqrt{4 \pi}} \int_{0}^{\tau} \frac{\mu\left(\tau^{\prime}\right)}{\left(\tau-\tau^{\prime}\right)^{3 / 2}} e^{-\frac{t^{2}}{4\left(\tau-\tau^{\prime}\right)}} d \tau^{\prime} .
\end{aligned}
$$

Our definition of the action of operator $e_{D, \mu}^{\lambda \partial_{t}^{2}}$ with any function $\mu$ can be presented as

$$
e_{D, J}^{\tau \partial_{t}^{2}} \varphi(t)=e_{0}^{\tau \partial_{t}^{2}} \varphi(t)+J(t, \tau)
$$

where

$$
e_{D, 0}^{\tau \partial_{t}^{2}} \varphi=\frac{1}{2 \sqrt{\tau \pi}} \int_{0}^{\infty} d t^{\prime}\left[e^{-\frac{\left(t-t^{\prime}\right)^{2}}{4 \tau}}-e^{-\frac{\left(t+t^{\prime}\right)^{2}}{4 \tau}}\right] \varphi\left(t^{\prime}\right)
$$

and the source $J$ is given by

$$
J(t, \tau)=\frac{t}{\sqrt{4 \pi}} \int_{0}^{\tau} \frac{\mu\left(\tau^{\prime}\right)}{\left(\tau-\tau^{\prime}\right)^{3 / 2}} e^{-\frac{t^{2}}{4\left(\tau-\tau^{\prime}\right)}} d \tau^{\prime} .
$$

Here the subscript $D$ means the Dirichlet and we write the index $J$ to stress that the definition depends on the choice of the daemon source $J$.

Consider also the Neumann problem for the heat equation on the half-line $t>0$ :

$$
\left\{\begin{array}{l}
\frac{\partial}{\partial \tau} \Psi_{N}(t, \tau)=\frac{\partial^{2}}{\partial t^{2}} \Psi_{N}(t, \tau), \quad t>0, \quad \tau>0, \\
\Psi_{N}(t, 0)=\varphi(t) \\
\left.\frac{\partial}{\partial t} \Psi_{N}(t, \tau)\right|_{t=0}=\nu(\tau) .
\end{array}\right.
$$

To solve the Neumann problem on the half-line one extends $\varphi$ to the whole line in such a way that the extension is even and gets the solution:

$$
\begin{aligned}
\Psi_{N}(t, \tau) & =\frac{1}{\sqrt{4 \pi \tau}} \int_{0}^{\infty} \varphi\left(t^{\prime}\right)\left[e^{-\frac{\left(t-t^{\prime}\right)^{2}}{4 \tau}}+e^{-\frac{\left(t+t^{\prime}\right)^{2}}{4 \tau}}\right] d t^{\prime} \\
& -\frac{1}{\sqrt{\pi}} \int_{0}^{\tau} \frac{\nu\left(\tau^{\prime}\right)}{\left(\tau-\tau^{\prime}\right)^{1 / 2}} e^{-\frac{t^{2}}{4\left(\tau-\tau^{\prime}\right)}} d \tau^{\prime} .
\end{aligned}
$$


We define the operator $e^{\tau \partial_{t}^{2}}$ on the half-line with the Neumann boundary condition as

$$
e_{N, J}^{\tau \partial_{t}^{2}} \varphi(t)=\Psi_{N}(t, \tau) .
$$

Here $N$ means the Neumann boundary condition and the source $J$ is given by

$$
J(t, \tau)=\frac{1}{\sqrt{\pi}} \int_{0}^{\tau} \frac{\nu\left(\tau^{\prime}\right)}{\left(\tau-\tau^{\prime}\right)^{1 / 2}} e^{-\frac{t^{2}}{4\left(\tau-\tau^{\prime}\right)}} d \tau^{\prime}
$$

with the daemon function $\nu$.

There are also other initial-boundary problems for the heat equation on the half-line but we will not discuss them in this paper.

\section{Laplace Daemon}

In this section we show that the definition of the operator $e^{\partial_{t}^{2}}$ on the half-line based on the Laplace transform is a special case of our definition (4.3) based on the solution of the heat equation with mixed boundary conditions.

The Laplace transform method for the definition of the operator $e^{\partial_{t}^{2}}$ on the halfline is used in [22], see Appendix 1 for more details.

We apply the general definition (A.7) to the case of exponential function and obtain the following definition

$$
e_{\{L\}}^{\tau \partial_{t}^{2}} \varphi(t)=\frac{1}{2 \pi i} \int_{c-i \infty}^{c+i \infty} d s e^{s t}\left[e^{\tau s^{2}} \tilde{\varphi}(s)-\sum_{k=1}^{\infty} \sum_{j=1}^{2 k} \frac{\tau^{k} s^{2 k-j}}{k !} \varphi^{(j-1)}(0)\right] .
$$

Here the subscript $L$ indicates that the definition is based on the Laplace transform.

Now we show that the definition (5.1) based on the Laplace transform is a particular case of the definition (4.3) based on the mixed problem for the heat equation with the special choice of the function $\mu$.

Proposition 1. The following relation takes place

$$
e_{\{L\}}^{\tau \partial_{t}^{2}} \varphi(t)=e_{D, J}^{\tau \partial_{t}^{2}} \varphi(t)
$$

where $J$ is

$$
J(t, \tau)=\frac{t}{4 \sqrt{\pi}} \int_{0}^{\tau} d \tau^{\prime} e^{-\frac{1}{4} \frac{t^{2}}{\left(\tau-\tau^{\prime}\right)}} \frac{\phi\left(\sqrt{\tau^{\prime}}\right)+\phi\left(-\sqrt{\tau^{\prime}}\right)}{\left(\tau-\tau^{\prime}\right)^{3 / 2}}
$$

and $\phi(x)$ is defined by a map

$$
\varphi(x) \rightarrow \phi(x)
$$

by using the following rule. If the function $\varphi(x)$ is given by the series

$$
\varphi(x)=\sum_{n=0}^{\infty} \varphi_{n} \frac{x^{n}}{n !}
$$


then

$$
\phi(x)=\sum_{n=0}^{\infty} \varphi_{n} \frac{x^{n}}{\left[\frac{n}{2}\right] !} .
$$

Formula (5.9) can be written more explicitly,

$$
\begin{aligned}
e_{\{L\}}^{\tau \partial_{t}^{2}} \varphi(t) & =\frac{1}{2 \sqrt{\tau \pi}} \int_{0}^{\infty} d t^{\prime}\left[e^{-\frac{\left(t-t^{\prime}\right)^{2}}{4 \tau}}-e^{-\frac{\left(t+t^{\prime}\right)^{2}}{4 \tau}}\right] \varphi\left(t^{\prime}\right) \\
& +\frac{t}{4 \sqrt{\pi}} \int_{0}^{\tau} d \tau^{\prime} e^{-\frac{1}{4} \frac{t^{2}}{\left(\tau-\tau^{\prime}\right)}} \frac{\phi\left(\sqrt{\tau^{\prime}}\right)+\phi\left(-\sqrt{\tau^{\prime}}\right)}{\left(\tau-\tau^{\prime}\right)^{3 / 2}} .
\end{aligned}
$$

This proposition shows that the definition of the operator $e_{L}^{\tau \partial_{t}^{2}}$ which uses the Laplace transform is a particular case of the definition of the operator $e_{\{\mu\}}^{\tau \partial_{t}^{2}}$ which uses the solution of the heat equation with the special daemon function $\mu$.

!!The proof of Proposition 1 is given in Appendix B.!! !!So, one can say that Laplace daemon is subordinated to Dirichlet's daemon.!!

\section{Solutions to Linearized Nonlocal Equation on the Half-line}

\subsection{Dirichlet's daemon without source}

Here we first consider the case of the Dirichlet's daemon with $j(\mu \mid t, \tau)=0$. In this case the equation of motion

$$
e_{D, 0}^{\tau \partial_{t}^{2}} \Phi(t)=m^{2} \Phi(t), \quad t>0
$$

where $m>0$ and $\tau>0$ are fixed parameters, has the form of the following integral equation

$$
\frac{1}{2 \sqrt{\tau \pi}} \int_{0}^{\infty} d t^{\prime}\left[e^{-\frac{\left(t-t^{\prime}\right)^{2}}{4 \tau}}-e^{-\frac{\left(t+t^{\prime}\right)^{2}}{4 \tau}}\right] \Phi\left(t^{\prime}\right)=m^{2} \Phi(t), \quad t>0 .
$$

One can solve equation (6.1) by making the odd extension of the function $\Phi(t)$ to the whole real line. Then we get the equation

$$
e^{\tau \partial_{t}^{2}} \Phi(t)=m^{2} \Phi(t), \quad t \in \mathbb{R}
$$

The odd solution of Eq. (6.3) is given by a linear combination

$$
\Phi_{D}(t)=\sum_{n}\left(\frac{1}{2} B_{n}\left(e^{\alpha_{n} t}-e^{-\alpha_{n} t}\right)+\frac{1}{2} B_{n}^{*}\left(e^{\alpha_{n}^{*} t}-e^{-\alpha_{n}^{*} t}\right)\right),
$$

where $\alpha_{n}$ are solutions to the equation

$$
e^{\tau \alpha_{n}^{2}}=m^{2}
$$




$$
\alpha_{n}= \pm \sqrt{\frac{\ln m^{2}+2 \pi i n}{\tau}}, n=0, \pm 1, \pm 2, \ldots
$$

For the $\alpha_{0}$ mode,

$$
\Phi(t)=B_{0} \sinh \alpha_{0} t
$$

we have

$$
e_{D, 0}^{\tau \partial_{t}^{2}} \sinh r t=e^{\tau r^{2}} \sinh r t, \quad t>0, \quad e^{\tau r^{2}}=m^{2}
$$

Indeed, the action of the operator $e_{0}^{\tau \partial_{t}^{2}}$ on the function $e^{r t}$ is given by the formula

$$
e_{D, 0}^{\tau \partial_{t}^{2}} e^{r t}=\frac{1}{2} e^{r t+r^{2} \tau}\left[1+\operatorname{erf}\left(r \sqrt{\tau}+\frac{t}{2 \sqrt{\tau}}\right)\right]-\frac{1}{2} e^{-r t+r^{2} \tau}\left[1+\operatorname{erf}\left(r \sqrt{\tau}-\frac{t}{2 \sqrt{\tau}}\right)\right]
$$

since

$$
\begin{aligned}
& \frac{1}{2 \sqrt{\tau \pi}} \int_{0}^{\infty} d t^{\prime}\left[e^{-\frac{\left(t-t^{\prime}\right)^{2}}{4 \tau}}-e^{-\frac{\left(t+t^{\prime}\right)^{2}}{4 \tau}}\right] e^{r t^{\prime}} \\
= & \frac{1}{2} e^{r t+r^{2} \tau}\left[1+\operatorname{erf}\left(r \sqrt{\tau}+\frac{t}{2 \sqrt{\tau}}\right)\right]-\frac{1}{2} e^{-r t+r^{2} \tau}\left[1+\operatorname{erf}\left(r \sqrt{\tau}-\frac{t}{2 \sqrt{\tau}}\right)\right]
\end{aligned}
$$

Hence, we get (6.8).

The energy and pressure on the solution have the form 16]

$$
\begin{aligned}
& E=-\frac{m^{2} \ln m^{2}}{2} B_{0}^{2} \\
& p=-\frac{m^{2} \ln m^{2}}{2} e^{2 \alpha_{0}^{2}} B_{0}^{2} \cosh \left(2 \alpha_{0} t\right)
\end{aligned}
$$

where we set $\tau=2$ and $g_{p}=1$. The energy is constant but the pressure depends on time. We see that if $m^{2}>1$ the energy $E$ is negative, but $-(E+p)$ that defines the derivative of the Hubble parameter (see Sect. 7) $\dot{H}$ is positive,

$$
\dot{H}=-\frac{1}{2 m_{p}^{2}}(E+p)=\frac{m^{2}}{2 m_{p}^{2}} \frac{\ln m^{2}}{2} B_{0}^{2}\left(1+\cosh \left(2 \alpha_{0} t\right)\right)
$$

Note that in the naive local approximation to (2.2) we deal with a massive ghost field and an appearance of a negative energy is not surprising. If we take in (2.2) $g_{p}=-1$ we see that the local approximation corresponds to the usual massive field and in this case we get the positive energy.

We will see in the next subsection that in the case of the Neunmann daemon we get the opposite signs. 


\subsection{Dirichlet's daemon with source}

Now we consider the nonlocal equation with the Dirichlet boundary conditions and with an external source

$$
e_{D, J}^{\tau \partial_{t}^{2}} \Phi=m^{2} \Phi
$$

We are going to find a solution to this equation for a special form of the daemon source $J$.

The action of the operator $e_{D, 0}^{\tau \partial_{t}^{2}}$ on the constant $C$ is given by

$$
e_{D, 0}^{\tau \partial_{t}^{2}} C=C \operatorname{erf}\left(\frac{1}{2} \frac{t}{\sqrt{\tau}}\right)
$$

where $\operatorname{erf}(t)$ is the error function, $(\mathbb{B} .29)$.

Acting by $e_{D, 0}^{\tau \partial_{t}^{2}}$ to $\Phi_{0}+B_{0} \sinh (r t)$ we get

$$
e_{D, 0}^{\tau \partial_{t}^{2}}\left(\Phi_{0}+B_{0} \sinh (r t)\right)=\Phi_{0} \operatorname{erf}\left(\frac{1}{2} \frac{t}{\sqrt{\tau}}\right)+B_{0} e^{\tau r^{2}} \sinh (r t)
$$

This relation we can interpret as

$$
e_{D, 0}^{\tau \partial_{t}^{2}} \Phi=-J(\tau, t)+m^{2} \Phi
$$

for $J(\tau, t)$ and $\Phi(t)$ in the form

$$
\begin{aligned}
J(\tau, t) & =m^{2} \Phi_{0}-\Phi_{0} \operatorname{erf}\left(\frac{1}{2} \frac{t}{\sqrt{\tau}}\right), \\
\Phi(t) & =\Phi_{0}+B_{0} \sinh (r t),
\end{aligned}
$$

and

$$
r^{2}=\frac{\ln m^{2}}{\tau} .
$$

According to (4.4) we rewrite (6.16) as (6.13).

\subsection{Neumann's daemon without source}

Here we consider the case of the Neumann daemon with $J=0$. In this case solutions to the linearized equation of motion

$$
e_{N, 0}^{\tau \partial_{t}^{2}} \Phi=m^{2} \Phi
$$

is given by a linear combination

$$
\Phi(t)=\sum_{n}\left(\frac{1}{2} C_{n}\left(e^{\alpha_{n} t}+e^{-\alpha_{n} t}\right)+\frac{1}{2} C_{n}^{*}\left(e^{\alpha_{n}^{*} t}+e^{-\alpha_{n}^{*} t}\right)\right)
$$

where $\alpha_{n}$ are given by equation (6.6). For the $\alpha_{0}$ mode

$$
\Phi(t)=C_{0} \cosh \alpha_{0} t
$$


we have

$$
e_{D, 0}^{\tau \partial_{t}^{2}} \cosh \alpha_{0} t=e^{\tau \alpha_{0}^{2}} \cosh \alpha_{0} t, \quad t>0
$$

The energy and pressure on this one mode approximation are 16]

$$
\begin{aligned}
& E_{N}=\frac{m^{2} \ln m^{2}}{2} C_{0}^{2} \\
& p_{N}=-\frac{m^{2} \ln m^{2}}{2} C_{0}^{2} \cosh \left(2 \alpha_{0} t\right) .
\end{aligned}
$$

We see that if $m^{2}>1$ the energy $E$ is positive as well as $-(E+p)$, and consequently the derivative of the Hubble parameter $\dot{H}$ is positive,

$$
\dot{H}=-\frac{1}{2 m_{p}^{2}}(E+p)=\frac{m^{2}}{2 m_{p}^{2}} \frac{\ln m^{2}}{2} C_{0}^{2}\left(-1+\cosh \left(2 \alpha_{0} t\right)\right) .
$$

This result is rather surprising, since we see that the energy is positive in the case corresponding to the case of the naive massive ghost mode.

\section{Nonlinear Tachyon Equation on the Whole Line}

It is instructive to compare the approximate solution obtained in the next section for the nonlocal equation on the half-line with the solution for the nonlinear tachyon equation on the whole line:

$$
e^{\tau \partial^{2}}\left(\xi^{2} \partial^{2}-\mu^{2}\right) \Phi(t)=-\epsilon \Phi^{3}(t), \quad \tau, \mu, \epsilon>0 .
$$

This equation on the whole line can be written as an integral equation

$$
\frac{1}{2 \sqrt{\tau \pi}} \int_{-\infty}^{\infty} d t^{\prime}\left(\frac{\xi^{2}\left(\left(t-t^{\prime}\right)^{2}-2 \tau\right)}{4 \tau^{2}}-\mu^{2}\right) e^{-\frac{\left(t-t^{\prime}\right)^{2}}{4 \tau}} \Phi\left(t^{\prime}\right)=-\epsilon \Phi^{3}(t) .
$$

This equation for $\xi=0$ an an odd function $\Phi(-t)=-\Phi(t)$ has the form

$$
\frac{1}{2 \sqrt{\tau \pi}} \int_{0}^{\infty} d t^{\prime}\left(e^{-\frac{\left(t-t^{\prime}\right)^{2}}{4 \tau}}-e^{-\frac{\left(t+t^{\prime}\right)^{2}}{4 \tau}}\right) \Phi\left(t^{\prime}\right)=\epsilon \Phi^{3}(t) .
$$

It is known [56] that this equation has a solution interpolating between two vacua $\Phi= \pm \frac{\mu}{\sqrt{\epsilon}}$.

It is also known 69 that for $\xi>\xi_{c r}$ equation (7.1) has oscillation solutions with period $T_{\xi}$ and within one period a good approximation for these solutions is

$$
\Phi(t)=a \sinh (\Omega t)-\frac{\epsilon a^{3}}{32 \mu^{2}} e^{-9 \lambda \Omega^{2}} \sinh (3 \Omega t)+\ldots
$$


where $\Omega$ is a root of

$$
\Omega^{2}-\frac{\mu^{2}}{\xi^{2}}-\frac{3}{4} \frac{\epsilon}{\xi^{2}} a^{2} e^{-\lambda \Omega^{2}}=0 .
$$

Approximation (7.4) is valid for small $\lambda$ and small $t, t<T_{\xi} / 4$. Solutions to (7.5) are given by the Lambert $\mathrm{W}$ function satisfying a relation $W(x) e^{W(x)}=x$,

$$
\Omega= \pm \sqrt{\frac{\mu^{2}}{\xi^{2}}+\frac{1}{\lambda} \mathrm{W}\left(\frac{3 \lambda \epsilon a^{2}}{4 \xi^{2}} e^{-\frac{\mu^{2}}{\xi^{2}} \lambda}\right)}
$$

Expanding the right hand site of (7.6) on $\lambda$ we get

$$
\Omega= \pm\left(\sqrt{\frac{\mu^{2}}{\xi^{2}}+\frac{3}{4} \frac{\epsilon}{\xi^{2}} a^{2}}-\frac{3}{8} \frac{\epsilon}{\xi^{2}} a^{2} \lambda \sqrt{\frac{\mu^{2}}{\xi^{2}}+\frac{3}{4} \frac{\epsilon}{\xi^{2}} a^{2}}+\mathcal{O}\left(\lambda^{2}\right)\right)
$$

Note, that if $\lambda=0$ one gets an approximate solution to the local equation

$$
\begin{gathered}
\left(\xi^{2} \partial^{2}-\mu^{2}\right) q(t)=-\epsilon q^{3}(t) \\
q(t)=A \sinh \left(\frac{\mu^{2}}{\xi^{2}}\left(t-\frac{3 A^{2}}{8 \mu^{2}} \epsilon t\right)\right)+\frac{\epsilon A^{3}}{32 \mu^{2}} \sinh \left(\frac{3 \mu^{2}}{\xi^{2}}\left(t-\frac{3 A^{2}}{8 \mu^{2}} \epsilon t\right)\right)+\ldots
\end{gathered}
$$

Note an appearance of the hyperbolic functions instead of the trigonometric functions in the Bogolyubov-Krylov averaging method for the nonlinear oscillations [76].

\section{Nonlinear Nonlocal Equations on the Half-line}

\subsection{Dirichlet's case}

In this section we consider a nonlinear nonlocal equation on the half-line of the following form

$$
e_{D, J}^{\tau \partial_{t}^{2}} \Phi=V^{\prime}(\Phi(t)),
$$

where $e_{D, J}^{\tau \partial_{t}^{2}}$ is given by (4.4). By taking $t=0$ in (8.1) we obtain

$$
\mu(\tau)=V^{\prime}(\Phi(0))
$$

Equation (8.2) shows that the initial value of the field $\Phi(0)$ is governed by the Dirichlet daemon function $\mu$.

Let us find an approximate solution to (8.1) for $V(\Phi)=\frac{g}{3} \Phi^{3}$. We consider the equation

$$
e_{D, 0}^{\tau \partial_{t}^{2}} \Phi=-J+g \Phi^{2}
$$

for $J$ in the form

$$
J(\tau, t)=\mu_{0}+\mu_{1} \operatorname{erf}\left(\frac{1}{2} \frac{t}{\sqrt{\tau}}\right) .
$$


We make the following expansion for $\Phi$

$$
\Phi(t)=B_{0}+B_{0} \sinh (r t)+B_{2} \sinh (2 r t)+\ldots \ldots
$$

and solve (8.3) term by term. We have

$$
\begin{aligned}
& e_{0}^{\tau \partial_{t}^{2}}\left(B_{0}+B_{0} \sinh (r t)+B_{2} \sinh (2 r t)+\ldots\right) \\
= & -\mu_{0}-\mu_{1} \operatorname{erf}\left(\frac{1}{2} \frac{t}{\sqrt{\tau}}\right)+g\left(B_{0}+B_{1} \sinh (r t)+B_{2} \sinh (2 r t)+\ldots\right)^{2} .
\end{aligned}
$$

Comparing terms with erf and $\sinh (r t)$ we get the set of relations

$$
\begin{aligned}
B_{0} & =\mu_{1}, \quad \quad \mu_{0}=B_{0}-\frac{g B_{1}^{2}}{2} . \\
e^{\lambda r^{2}} B_{1} & =2 g B_{0}^{2} B_{1}, \quad e^{\lambda 4 r^{2}} B_{1}=\frac{g B_{1}^{2}}{2}+2 g B_{0} B_{2}
\end{aligned}
$$

that gives

$$
\begin{array}{ll}
B_{0}=\mu_{1}, & B_{1}= \pm \sqrt{\frac{2\left(\mu_{0}+\mu_{1}\right)}{g}}, \\
r^{2}=\frac{\ln \left(2 g \mu_{1}^{2}\right)}{\lambda}, & B_{2}=\frac{1}{2 g \mu_{1}}\left(\mu_{0}+\mu_{1} \mp\left(2 g \mu_{1}^{2}\right)^{4} \sqrt{\frac{2\left(\mu_{0}+\mu_{1}\right)}{g}}\right) .
\end{array}
$$

\subsection{Neumann's case}

In this section we consider

$$
\left(-\xi^{2} \partial_{t}^{2}+m^{2}\right) e_{N J}^{\lambda \partial_{t}^{2}} \Phi=V^{\prime}(\Phi)
$$

Taking into account (4.8) we get

$$
\begin{aligned}
\left(-\xi^{2} \partial_{t}^{2}+m^{2}\right) e_{N 0}^{\lambda \partial_{t}^{2}} \Phi & =V^{\prime}(\Phi)-J_{\xi} \\
J_{\xi} & =\left(-\xi^{2} \partial_{t}^{2}+m^{2}\right) J
\end{aligned}
$$

where the source $J$ is given by (4.10). Assuming that $J$ admits an expansion

$$
J_{N S F T}(t)=g j_{0}+g j_{1} \cosh (\Omega t)+g j_{2} \cosh (2 \Omega t)+\ldots \ldots
$$

(with some still unknown $\Omega$ ) we make the similar expansion for $\Phi$

$$
\Phi(t)=B_{0}+B_{1} \cosh (\Omega t)+B_{2} \cosh (2 \Omega t)+\ldots \ldots
$$

and solve (8.3) term by term. We get

$$
\begin{aligned}
& \Omega^{2}=\frac{m^{2}}{\xi^{2}}+\left(\frac{g j_{1}}{\xi^{2} B_{1}}+\frac{2 g^{2} j_{0}}{\xi^{2} m^{2}}-\frac{g^{2} B_{1}^{2}}{\xi^{2} m^{2}}\right) e^{\tau \Omega^{2}} \\
& B_{0}=-\frac{g j_{0}}{m^{2}}+\frac{1}{2} \frac{g B_{1}^{2}}{m^{2}} \\
& B_{2}=\frac{g j_{2}}{4 \xi^{2} \Omega^{2}-m^{2}} e^{-4 \tau \Omega^{2}}
\end{aligned}
$$


and so on. Equation (8.17) defines $\Omega^{2}$ perturbatively,

$$
\Omega^{2}=\frac{m^{2}}{\xi^{2}}+\frac{g j_{1}}{\xi^{2} B_{1}} e^{\frac{\tau m^{2}}{\xi^{2}}}+\ldots
$$

and the last equations define perturbatively coefficients. We can see that this perturbative series is similar to the local perturbation series for the equation

$$
\left(-\xi^{2} \partial_{t}^{2}+m^{2}\right) \Phi=-g J+g \Phi^{2}
$$

It is known that equation 8.21) exhibits very interesting chaotic behavior for special currents, that cannot be seen perturbatively. We will mention about these properties in Sect. 11.

\section{Nonlocal Equation on the Half-line in Friedmann Cosmol- ogy}

For simplicity we consider the action 2.3 with $\xi=0$ :

$$
S=\frac{1}{g_{4}} \int d^{4} x \sqrt{-g}\left\{m_{p}^{2} \frac{R}{2}+\frac{1}{\gamma}\left(\frac{1}{2} \Phi e^{-\frac{1}{4} \square} \Phi-V(\Phi)\right)\right\} .
$$

Here all coordinates are dimensionless, see notations in Sect.2, $x \rightarrow x / M_{s}, m_{p}^{2}=$ $M_{p}^{2} / M_{s}^{2}, \gamma$ is dimensionless coupling constant.

In the spatially flat Friedmann metric

$$
d s^{2}=-d t^{2}+a^{2}(t) d x^{2}
$$

the dynamics in the model is described by a system of two nonlinear nonlocal equations [?] for the tachyon field and the Hubble parameter $H(t)=\dot{a} / a$

$$
\begin{aligned}
e^{-\frac{1}{4} \mathcal{D}} \Phi & =V^{\prime}(\Phi), t>0 \\
3 H^{2} & =\frac{1}{\gamma m_{p}^{2}}\left(\frac{1}{2} \Phi e^{-\frac{1}{4} \mathcal{D}} \Phi+V(\Phi)+\mathcal{E}_{1}+\mathcal{E}_{2}\right),
\end{aligned}
$$

where

$$
\mathcal{D}=-\partial_{t}^{2}-3 H(t) \partial_{t}, \quad H=\frac{\partial_{t} a}{a}
$$

and

$$
\begin{aligned}
& \mathcal{E}_{1}=\frac{1}{8} \int_{0}^{1} d s\left(e^{\frac{s-2}{8} \mathcal{D}} \Phi\right) \cdot\left(\mathcal{D} e^{-\frac{1}{8} s \mathcal{D}} \Phi\right) \\
& \mathcal{E}_{2}=\frac{1}{8} \int_{0}^{1} d s\left(\partial_{t} e^{\frac{s-2}{8} \mathcal{D}} \Phi\right) \cdot\left(\partial_{t} e^{-\frac{1}{8} s \mathcal{D}} \Phi\right)
\end{aligned}
$$

The nonlocal energy $\mathcal{E}_{1}$ plays the role of an extra potential term and $\mathcal{E}_{2}$ the role of the kinetic term. 


\subsection{Dirichlet's case}

Let us define the action of the operator $e_{D, J}^{-\tau \mathcal{D}}$ (compare with the definition of the same operator on the whole axis used in [21, 37, 32]) on the function $\varphi(t)$ as

$$
e_{D, J}^{-\tau \mathcal{D}} \varphi(t)=\Psi_{D, H}(t, \tau),
$$

where $\Psi_{D, H}(t, \tau)$ is a solution to

$$
\left\{\begin{array}{l}
\frac{\partial}{\partial \tau} \Psi_{D, H}(t, \tau)=-\mathcal{D} \Psi_{D, H}(t, \tau), \quad t>0, \quad \tau>0, \\
\Psi_{D, H}(t, 0)=\varphi(t), \\
\Psi_{D, H}(0, \tau)=\mu(\tau) .
\end{array}\right.
$$

Let us consider the case $H(t)=H_{0}=$ constant. Note that solutions to the first equations in (9.9) and (4.1) are related via

$$
\Psi_{D, H_{0}}(t, \tau)=e^{-\frac{3}{2} H_{0} t-\frac{9}{4} H_{0}^{2} \tau} \Psi_{D}(t, \tau) .
$$

Now we can write the solution to (9.9) for $H(t)=H_{0}$ in the form

$$
\begin{aligned}
\Psi_{D, H_{0}}(t, \tau) & =e^{-\frac{3}{2} H_{0} t-\frac{9}{4} H_{0}^{2} \tau}\left[\frac{1}{\sqrt{4 \pi \tau}} \int_{0}^{\infty} e^{\frac{3}{2} H_{0} t^{\prime}} \varphi\left(t^{\prime}\right)\left[e^{-\frac{\left(t-t^{\prime}\right)^{2}}{4 \tau}}-e^{-\frac{\left(t+t^{\prime}\right)^{2}}{4 \tau}}\right] d t^{\prime}\right. \\
& \left.+\frac{t}{\sqrt{4 \pi}} \int_{0}^{\tau} \frac{e^{\frac{9}{4} H_{0}^{2} \tau^{\prime}} \mu\left(\tau^{\prime}\right)}{\left(\tau-\tau^{\prime}\right)^{3 / 2}} e^{-\frac{t^{2}}{4\left(\tau-\tau^{\prime}\right)}} d \tau^{\prime}\right] .
\end{aligned}
$$

Denoting $\Psi_{D, H_{0}}(t, \tau)=e_{D, \mathcal{J}}^{-\tau \mathcal{D}} \varphi(t)$ we can write

$$
e_{N, \mathcal{J}}^{-\tau \mathcal{D}} \varphi(t)=e_{N, 0}^{-\tau \mathcal{D}} \varphi(t)+\mathcal{J}(\tau, t),
$$

where

$$
\mathcal{J}(\tau, t)=e^{-\frac{3}{2} H_{0} t-\frac{9}{4} H_{0}^{2} \tau} \frac{t}{\sqrt{4 \pi}} \int_{0}^{\tau} \frac{e^{\frac{9}{4} H_{0}^{2} \tau^{\prime}} \mu\left(\tau^{\prime}\right)}{\left(\tau-\tau^{\prime}\right)^{3 / 2}} e^{-\frac{t^{2}}{4\left(\tau-\tau^{\prime}\right)}} d \tau^{\prime} .
$$

The action of operator $e_{D, 0}^{-\tau \mathcal{D}}$ on the function $e^{-\frac{3}{2} H_{0} t} \sinh (r t)$ is given by the formula

$$
e_{D, 0}^{-\tau \mathcal{D}} e^{-\frac{3}{2} H_{0} t} \sinh (r t)=e^{\tau\left(r^{2}-\frac{9}{4} H_{0}^{2}\right)} e^{-\frac{3}{2} H_{0} t} \sinh (r t) .
$$

\subsection{Neumann's case}

In an analogous way we define the action of the Neunmann operator $e_{N, \mu}^{-\tau \mathcal{D}}$ on the function $\varphi(t)$ as

$$
e_{N, \nu}^{-\tau \mathcal{D}} \varphi(t)=\Psi_{N, H}(t, \tau),
$$

where $\Psi_{N, H}(t, \tau)$ is the solution to

$$
\left\{\begin{array}{l}
\frac{\partial}{\partial \tau} \Psi_{N, H}(t, \tau)=-\mathcal{D} \Psi_{N, H}(t, \tau), \quad t>0, \quad \tau>0, \\
\Psi_{N, H}(t, 0)=\varphi(t), \\
\left.\frac{\partial}{\partial t} \Psi_{N, H}(t, \tau)\right|_{t=0}=\nu(\tau),
\end{array}\right.
$$


For $H(t)=H_{0}$ the solution of (9.16) has the form

$$
\begin{aligned}
\Psi_{N, H_{0}}(t, \tau) & =e^{-3 H_{0} t-\frac{9}{4} H_{0}^{2} \tau}\left[\frac{1}{\sqrt{4 \pi \tau}} \int_{0}^{\infty} e^{3 H_{0} t^{\prime}} \varphi\left(t^{\prime}\right)\left[e^{-\frac{\left(t-t^{\prime}\right)^{2}}{4 \tau}}+e^{-\frac{\left(t+t^{\prime}\right)^{2}}{4 \tau}}\right] d t^{\prime}\right. \\
& \left.+\frac{1}{\sqrt{\pi}} \int_{0}^{\tau} \frac{e^{\frac{9}{4} H_{0}^{2} \tau^{\prime}} \nu\left(\tau^{\prime}\right)}{\left(\tau-\tau^{\prime}\right)^{1 / 2}} e^{-\frac{t^{2}}{4\left(\tau-\tau^{\prime}\right)}} d \tau^{\prime}\right]
\end{aligned}
$$

The action of the operator $e_{N, 0}^{-\tau \mathcal{D}}$ on the function $e^{-\frac{3}{2} H_{0} t} \cosh (r t)$ is given by

$$
e_{N, 0}^{-\tau \mathcal{D}} e^{-\frac{3}{2} H_{0} t} \cosh (r t)=e^{\tau\left(r^{2}-\frac{9}{4} H_{0}^{2}\right)} e^{-\frac{3}{2} H_{0} t} \cosh (r t)
$$

\subsection{Solutions to Linearized Nonlocal Equation on the Half-line, $H_{0} \neq 0$}

Here we consider the case of the Dirichlet daemon with $j(\mu \mid t, \tau)=0$. The equation of motion

$$
e_{D, 0}^{\tau \mathcal{D}} \Phi_{H_{0}}(t)=m^{2} \Phi_{H_{0}}(t), t>0, \tau>0,
$$

where $\tau>0$ is a fixed parameter, has the form of the following integral equation

$$
\frac{e^{-\frac{3}{2} H_{0} t-\frac{9}{4} H_{0}^{2} \tau}}{2 \sqrt{\tau \pi}} \int_{0}^{\infty} d t^{\prime} e^{\frac{3}{2} H_{0} t}\left[e^{-\frac{\left(t-t^{\prime}\right)^{2}}{4 \tau}}-e^{-\frac{\left(t+t^{\prime}\right)^{2}}{4 \tau}}\right] \Phi\left(t^{\prime}\right)=m^{2} \Phi(t), \quad t>0
$$

From (9.18) we see that

$$
\Phi_{H_{0}}(t)=B_{1} e^{-\frac{3}{2} H_{0} t} \sinh (r t)
$$

solves equation $(9.20)$ if

$$
\left(r^{2}-\frac{9}{4} H_{0}^{2}\right)=\frac{\ln m^{2}}{\tau}
$$

i.e. the spectrum of the operator $e_{D, 0}^{\tau \mathcal{D}_{H_{0}}}$ is the same as spectrum of the operator $e^{\tau \mathcal{D}_{H_{0}}}$ on the whole axis.

From this consideration we get that the energy and pressure

$$
\begin{aligned}
& E_{D, H_{0}}\left(B_{1}\right)=-\left(r^{2}-\frac{9}{4} H_{0}^{2}\right) e^{\left(r^{2}-\frac{9}{4} H_{0}^{2}\right)} B_{1}^{2}, \\
& P_{D, H_{0}}\left(B_{1}\right)=-\left(r^{2}-\frac{9}{4} H_{0}^{2}\right) B_{1}^{2} e^{\left(r^{2}-\frac{9}{4} H_{0}^{2}\right)} \cosh \left(2 t \sqrt{r^{2}-\frac{9}{4} H_{0}^{2}}\right) .
\end{aligned}
$$

This energy and pressure can be used to find a deviation of the FRW metric from the dS one.

\section{Inflation and External Sources}

We have obtained in this paper that in nonlocal string field theory equations on the half-line a new arbitrary function appears which was called the daemon external 
source. Let us now discuss a possible role of the external source in the cosmological inflation scenario.

The cosmological observations show that the universe is almost flat and a density perturbations are scale invariant, Gaussian and adiabatic. The simplest explanation of these observations is provided by the slow-roll inflation driven by a scalar field [77].

There are two versions of the inflation scenario with a single scalar field [1]-[团]. In the new inflation scenario one uses a large cosmological constant (the simplest potential is $U(\phi)=U_{0}-m^{2} \phi^{2} / 2$ ) while the chaotic inflation scenario works with the vanishing cosmological constant (the simplest potential is $U(\phi)=m^{2} \phi^{2} / 2$ ).

The basic ideas of the chaotic inflation scenario introduced by Linde [78, 79] are very natural and general. It is assumed that the early universe initially consisted of many domains with chaotically distributed scalar field. Then the rapid expansion (inflation) of the early universe made from the domains, in which the scalar field was rather large, many very big homogeneous domains. Our observable universe is one of these big homogeneous domains.

A great advantage of the chaotic inflation scenario is its simplicity: it may occur even in the theory of the free massive scalar field. The chaotic inflation occurs in many models of the scalar field with sufficiently flat potentials and for a wide class of randomly chosen initial conditions.

It is supposed that the natural initial conditions for the scalar field $\varphi_{0}$ at the moment when the classical description of the universe first becomes feasible is $U\left(\phi_{0}\right) \lesssim$ $M_{P}^{4}$, where $U(\phi)$ is the potential and $M_{P}$ is the Planck mass. If $U(\phi)=g \phi^{n}$, where $n>1,0<g<1$, then it follows that the typical initial value $\phi_{0}$ of the field $\phi$ in the early universe is large, $\phi_{0}>\left(M_{P}^{4} / g\right)^{1 / n}$, greater than the Planck mass.

One could wish to get an inflation scenario with smaller initial values of the field since at the Planck scale one doubts that the classical description is applicable. We will see that the external source can help to make smaller at least one of the slow roll parameters $(\epsilon)$ even for rather small initial values of the scalar.

Given this picture of chaotically distributed scalar field in the early universe we can ask ourself whether other dynamical and external fields and sources could change the conclusions of the chaotic inflation scenario? It seems that the basic result, that inflation created our modern homogeneous universe from a rather randomly chosen domain in the initial chaos, still should be valid in this more general situation with arbitrary number of chaotically distributed fields and sources.

Let us consider the action (1.6). The Friedmann equations for the homogeneous field $\phi=\phi(t)$ and the source $J=J(t)$ have the form

$$
\begin{gathered}
\ddot{\phi}+3 H \dot{\phi}+U^{\prime}(\phi)-J=0, \\
H^{2}=\frac{1}{3 M_{P}^{2}}\left(\frac{\dot{\phi}^{2}}{2}+U(\phi)-J \phi\right) .
\end{gathered}
$$


The slow roll parameters are

$$
\epsilon=\frac{M_{P}^{2}}{2}\left(\frac{U^{\prime}(\phi)-J}{U(\phi)-J \phi}\right)^{2}, \eta=M_{P}^{2} \frac{U^{\prime \prime}(\phi)}{U(\phi)-J \phi}
$$

If the potential $U(\phi)=m^{2} \phi^{2} / 2$ then

$$
\epsilon=\frac{M_{P}^{2}}{2 \phi^{2}} \frac{\delta^{2}}{\left(\frac{m^{2}}{2} \phi-\delta\right)^{2}}
$$

where we denote $\delta=m^{2} \phi-J$. Therefore in the spacetime domain, where the daemon source $J$ is such that $\delta \ll m^{2} \phi / 2$, we get a small parameter $\epsilon$ even for the not so large field $\phi$. We can not make the parameter $\eta$ very small for the small field $\phi$ by using a reasonable choice of the source $J$. To this end, we still have to take the large values of the field $\phi$ or the large cosmological constant. Note, that if the souse $J(t)$ in (10.1) has the form

$$
J(t)=\sum_{n} \epsilon_{n} \cos \left(\omega_{n} t+\theta_{n}\right)
$$

and there is no $J \phi$ term in $(10.2)$, one gets a nonlinear resonance 83]. The external sources play the role of the perturbation in the FRW cosmology. Now, the result of the action of this perturbation to the evolution of the Universe depends of the form of perturbation. If the source behaves like a monotonic function then the daemon source can help to get the chaotic inflation scenario with small scalar field. However, if the source is a periodic function, when one could get the nonlinear resonance which destroys the inflation scenario.

Note also that if the current $J(x)$ in (10.1) is a white noise with the correlation function

$$
\langle J(x) J(y)\rangle=H^{5} \delta(x-y)
$$

and if one can neglect the term $J \phi$ in then we obtain the Langevin equation in the stochastic approach to inflation introduced by Starobinsky [54, for a general discussion see [80]. The stochastic approach fits well with the functional approach to mechanics [81]. A general procedure of obtaining the quantum white noise from quantum field theory is developed in the stochastic limit framework [82].

According to the anthropic principle perhaps there are many universes and our universe is just one of them better suitable to support life as we know it. Actually this is just a phenomenological principle that the theory should be consistent with observations. Various local fields $\psi_{n}$ in (2.5) accompanied by the daemon sources could live in different universes developing dynamics needed for the inflation scenario. If there is a singularity also in future then it seems one has to set also a daemon boundary condition there. 


\section{Conclusion}

In this paper we have shown that string theory leads to nonlocal equations on the semi-axis which include an arbitrary source function $J(x)$. At the moment we go not have a theoretical principle which could help to fix $J(x)$ except a phenomenological requirement to be consistent with the observed universe.

There are many interesting mathematical and physical questions to be considered in the theory of the cosmological daemon. We hope to discuss them in future works.

\section{Acknowledgements}

We would like to thank Branko Dragovich, Alexey Koshelev and Sergey Vernov for the helpful discussions. The work is partially supported by grants RFFI 11-01-00894a, NS 8265.2010.1 (I.A.) and RFFI 11-01-00828-a, NS 7675.2010.1 (I.V.). 


\section{A. Laplace transformation and definition of $F\left(\partial_{t}\right)$}

We remind that the Laplace transform of the function $\varphi(t), t>0$ is defined as

$$
\tilde{\varphi}(s)=\int_{0}^{\infty} \varphi(t) e^{-s t} d t, \quad \operatorname{Re} s>a,
$$

where $a$ is some number. The inverse transform is given by

$$
\varphi(t)=\frac{1}{2 \pi i} \int_{c-i \infty}^{c+i \infty} \tilde{\varphi}(s) e^{t s} d s,
$$

where $c>a$.

The action of derivatives is given by 75

$$
\partial_{t}^{n} \phi(t)=\frac{1}{2 \pi i} \int_{c-i \infty}^{c+i \infty} d s e^{s t} s^{n}\left[\tilde{\phi}(s)-\sum_{j=0}^{n-1} \frac{\partial_{t}^{j} \phi(0)}{s^{j+1}}\right]
$$

Definition (A.3) has a meaning under the assumption that the function

$$
\Phi(z)=z^{n}\left[\tilde{\phi}(z)-\sum_{j=0}^{n-1} z^{-j-1} \partial_{t}^{j} \phi(0)\right]
$$

is holomorphic in $\operatorname{Re} z>a$ and satisfies condition

$$
|\Phi(z)|=\mathcal{O}\left(|z|^{-1-\alpha}\right), \text { for }|z| \rightarrow \infty, a \leq \operatorname{Re} z
$$

with $\alpha>0$ and $a \leq c$.

It follows from (A.3) that for an analytical function

$$
F(z)=\sum_{n=0}^{\infty} F_{n} z^{n} .
$$

it is natural to define the action of the operator $F\left(\partial_{t}\right)$ as [22]

$$
F\left(\partial_{t}\right) \varphi(t)=\frac{1}{2 \pi i} \int_{c-i \infty}^{c+i \infty} d s e^{s t}\{F(s) \tilde{\varphi}(s)-r(s)\},
$$

where

$$
r(s)=\sum_{n=0}^{\infty} \sum_{j=1}^{n} F_{n} s^{n-j} \partial_{t}^{j-1} \varphi(0) .
$$

!!Applying the general definition (A.7) to the case of exponential function we obtain definition (5.1) presented in the text.!! In 61] has been mentioned a more general definition of $F\left(\partial_{t}\right)$ that also uses the representation (A.7) but the residual term now is defined by infinite number of constants $d_{j}$

$$
r(s)=\sum_{n=0}^{\infty} \sum_{j=1}^{n} F_{n} d_{j-1} s^{n-j} .
$$


For the case of the $\exp \left(\tau \partial_{t}^{2}\right)$ one gets the following definition

$$
e_{\{L G\}}^{\tau \partial_{t}^{2}} \varphi(t)=\frac{1}{2 \pi i} \int_{c-i \infty}^{c+i \infty} d s e^{s t}\left[e^{\tau s^{2}} \tilde{\varphi}(s)-\sum_{k=1}^{\infty} \sum_{j=1}^{2 k} \frac{\tau^{k} s^{2 k-j}}{k !} d_{j-1}\right] .
$$

Here $d_{j}$ are arbitrary constant and the subscript $L G$ indicates that the definition is based on the modification of formula (5.1) based on the Laplace transform.

\section{B. Proof of proposition 1.}

To prove Proposition 1 we use the representation (5.1) and the following two lemmas.

Lemma 1. One has

$$
\frac{1}{2 \pi i} \int_{c-i \infty}^{c+i \infty} d s e^{s t} e^{\tau s^{2}} \tilde{\varphi}(s)=\frac{1}{2 \sqrt{\tau \pi}} \int_{0}^{\infty} d t^{\prime} e^{-\frac{\left(t-t^{\prime}\right)^{2}}{4 \tau}} \varphi\left(t^{\prime}\right),
$$

here $\tilde{\varphi}(s)$ is the Laplace transform of $\varphi(t)$.

Proof. Substituting (A.1) to the LHS of (B.1) we get

$$
\frac{1}{2 \pi i} \int_{c-i \infty}^{c+i \infty} d s e^{s t} e^{\tau s^{2}} \tilde{\varphi}(s)=\frac{1}{2 \pi i} \int_{c-i \infty}^{c+i \infty} d s e^{s t} e^{\tau s^{2}} \int_{0}^{\infty} \varphi\left(t^{\prime}\right) e^{-s t^{\prime}} d t^{\prime} .
$$

Assuming that we can interchange the order of integration and writing integral on $s$ as integral on $w, s=c+i w$ we can write

$$
\begin{gathered}
\frac{1}{2 \pi i} \int_{c-i \infty}^{c+i \infty} d s e^{s t} e^{\tau s^{2}} \int_{0}^{\infty} \varphi\left(t^{\prime}\right) e^{-s t^{\prime}} d t^{\prime}=\frac{1}{2 \pi} \int_{0}^{\infty} \int_{-\infty}^{\infty} d s e^{(c+i w)\left(t-t^{\prime}\right)} e^{\tau(c+i w)^{2}} \varphi\left(t^{\prime}\right) d t^{\prime} \\
=\frac{1}{2 \sqrt{\pi \tau}} \int_{0}^{\infty} e^{-\frac{\left(t-t^{\prime}\right)^{2}}{4 \tau}} \varphi\left(t^{\prime}\right) d t^{\prime}
\end{gathered}
$$

That proves the Lemma 1.

Lemma 2. The function $r(s, \tau)$ which is given by

$$
r(s, \tau)=\sum_{k=1}^{\infty} \sum_{j=1}^{2 k} \frac{\tau^{k} s^{2 k-j}}{k !} \varphi^{(j-1)}(0)
$$

admits the following representation

$$
r(s, \tau)=\int_{0}^{\tau} d \tau^{\prime} e^{s^{2}\left(\tau-\tau^{\prime}\right)}\left(\mathfrak{j}_{0}\left(\tau^{\prime}\right)+s \dot{j}_{1}\left(\tau^{\prime}\right)\right),
$$


where

$$
\begin{aligned}
& \mathfrak{j}_{0}(\tau)=\sum_{k=0}^{\infty} \frac{\tau^{k}}{k !} \lim _{t \rightarrow+0} \partial_{t}^{2 k+1} \varphi(t), \\
& \mathfrak{j}_{1}(\tau)=\sum_{k=0}^{\infty} \frac{y^{k}}{k !} \lim _{t \rightarrow+0} \partial_{t}^{2 k} \varphi(t) .
\end{aligned}
$$

Proof. Formula (B.4) can be rewritten in the following way

$$
\begin{aligned}
r(s, \tau)= & \sum_{k=0}^{\infty}\left[\varphi^{(2 k)}(0) \frac{e^{s^{2} \tau}-1-s^{2} \tau-\frac{1}{2 !}\left(s^{2} \tau\right)^{2}-\ldots-\frac{1}{k !}\left(s^{2} \tau\right)^{k}}{s^{2 k+1}}\right. \\
& \left.+\varphi^{(2 k+1)}(0) \frac{e^{s^{2} \tau}-1-s^{2} \tau-\frac{1}{2 !}\left(s^{2} \tau\right)^{2}-\ldots-\frac{1}{k !}\left(s^{2} \tau\right)^{k}}{s^{2 k+2}}\right] .
\end{aligned}
$$

Using

$$
e^{s^{2} \tau} \int_{0}^{\tau} e^{-s^{2} y} \frac{y^{m}}{m !} d y=\frac{e^{s^{2} \tau}-1-s^{2} \tau-\frac{1}{2}\left(s^{2} \tau\right)^{2}-\frac{1}{3 !}\left(s^{2} \tau\right)^{3} \ldots-\frac{1}{m !}\left(s^{2} \tau\right)^{m}}{s^{2 m+2}}
$$

we can rewrite $r(s, \tau)$ as

$$
\begin{aligned}
r(s, \tau) & =\sum_{k=0}^{\infty} e^{s^{2} \tau} \partial_{t}^{2 k} \varphi(0) s \int_{0}^{\tau} e^{-s^{2} y} \frac{y^{k}}{k !} d y \\
& +\sum_{k=0}^{\infty} e^{s^{2} \tau} \partial_{t}^{2 k+1} \varphi(0) \int_{0}^{\tau} e^{-s^{2} y} \frac{y^{k}}{k !} d y
\end{aligned}
$$

that gives the proof of Lemma 2 .

Lemma 3. The functions $\mathbf{j}_{0}$ and $\mathfrak{j}_{1}$, given by (B.0) and (B.X), admit the following representation

$$
\mathfrak{j}_{0}\left(\tau^{\prime}\right)=\frac{\phi\left(\sqrt{\tau^{\prime}}\right)-\phi\left(-\sqrt{\tau^{\prime}}\right)}{2 \sqrt{\tau^{\prime}}}, \mathfrak{j}_{1}\left(\tau^{\prime}\right)=\frac{\phi\left(\sqrt{\tau^{\prime}}\right)+\phi\left(-\sqrt{\tau^{\prime}}\right)}{2},
$$

where $\phi$ is related to $\varphi$ by (5.0).

Proof. Substituting into (B.10) the relations

$$
\phi_{n}=\frac{n !}{\left[\frac{n}{2}\right] !} \varphi_{n}
$$

we get the representation in terms of $\phi$

$$
\begin{aligned}
r(s, \tau) & =\sum_{k=0} e^{s^{2} \tau} \phi_{2 k} \int_{0}^{\tau} e^{-s^{2} y} \frac{s^{k} y^{2 k}}{(2 k) !} d y \\
& +\sum_{k=0} e^{s^{2} \tau} \phi_{2 k+1} \int_{0}^{\tau} e^{-s^{2} y} \frac{s^{k} y^{2 k+1}}{(2 k+1) !} d y .
\end{aligned}
$$


Taking into account that

$$
\sum_{k=0}^{\infty} \phi_{2 k+1} \frac{\tau^{k}}{(2 k+1) !}=\frac{\phi(\sqrt{\tau})-\phi(-\sqrt{\tau})}{2 \sqrt{\tau}}
$$

and

$$
\sum_{k=0}^{\infty} \phi_{2 k} \frac{\tau^{\frac{2 k}{2}}}{(2 k) !}=\frac{\phi(\sqrt{\tau})+\phi(-\sqrt{\tau})}{2}
$$

we get

$$
r(s, \tau)=\int_{0}^{\tau} d \tau^{\prime} e^{s^{2}\left(\tau-\tau^{\prime}\right)}\left[\frac{\phi\left(\sqrt{\tau^{\prime}}\right)-\phi\left(-\sqrt{\tau^{\prime}}\right)}{2 \sqrt{\tau^{\prime}}}+s \frac{\phi\left(\sqrt{\tau^{\prime}}\right)+\phi\left(-\sqrt{\tau^{\prime}}\right)}{2}\right],
$$

that proves Lemma 3 .

\section{Lemma 4.}

The second term in the integrand in the RHS of (5.1)

$$
r_{L}(t, \tau)=\frac{1}{2 \pi i} \int_{c-i \infty}^{c+i \infty} d s e^{s t} r(s, \tau)
$$

admits the following integral representation

$$
r_{L}(t, \tau)=\frac{1}{2 \sqrt{\pi}} \int_{0}^{\tau} d \tau^{\prime} e^{-\frac{1}{4} \frac{t^{2}}{\left(\tau-\tau^{\prime}\right)}} \frac{\mathfrak{j}_{0}\left(\tau^{\prime}\right)}{\sqrt{\tau-\tau^{\prime}}}-\frac{t}{4 \sqrt{\pi}} \int_{0}^{\tau} d \tau^{\prime} e^{-\frac{1}{4} \frac{t^{2}}{\left(\tau-\tau^{\prime}\right)}} \frac{\mathfrak{j}_{1}\left(\tau^{\prime}\right)}{\left(\tau-\tau^{\prime}\right)^{3 / 2}}(. \mathrm{B} .1
$$

The proof follows from the representation $(\mathbb{B} .16)$ by computing the Laplace transform. Indeed we have

$$
\begin{aligned}
r_{L}(t, \tau) & =\frac{1}{2 \pi i} \int_{c-i \infty}^{c+i \infty} d s e^{s t} r(s, \tau) \\
& =\frac{1}{2 \pi i} \int_{c-i \infty}^{c+i \infty} d s e^{s t} \int_{0}^{\tau} d \tau^{\prime} e^{s^{2}\left(\tau-\tau^{\prime}\right)} \mathfrak{j}\left(\tau^{\prime}, s\right) \\
& =\frac{1}{2 \pi i} \int_{c-i \infty}^{c+i \infty} d s e^{s t} \int_{0}^{\tau} d \tau^{\prime} e^{s^{2}\left(\tau-\tau^{\prime}\right)}\left[\mathfrak{j}_{0}\left(\tau^{\prime}\right)+s \dot{j}_{1}\left(\tau^{\prime}\right)\right] \\
& =\frac{1}{2 \sqrt{\pi}} \int_{0}^{\tau} d \tau^{\prime} e^{-\frac{1}{4} \frac{t^{2}}{\left(\tau-\tau^{\prime}\right)}} \frac{\mathfrak{j}_{0}\left(\tau^{\prime}\right)}{\sqrt{\tau-\tau^{\prime}}}-\frac{t}{4 \sqrt{\pi}} \int_{0}^{\tau} d \tau^{\prime} e^{-\frac{1}{4} \frac{t^{2}}{\left(\tau-\tau^{\prime}\right)}} \frac{\mathfrak{j}_{1}\left(\tau^{\prime}\right)}{\left(\tau-\tau^{\prime}\right)^{3 / 2}} .
\end{aligned}
$$

Therefore, we get

$$
r_{L}(t, \tau)=\frac{1}{2 \sqrt{\pi}} \int_{0}^{\tau} d \tau^{\prime} e^{-\frac{1}{4} \frac{t^{2}}{\left(\tau-\tau^{\prime}\right)}} \frac{\mathfrak{j}_{0}\left(\tau^{\prime}\right)}{\sqrt{\tau-\tau^{\prime}}}-\frac{t}{4 \sqrt{\pi}} \int_{0}^{\tau} d \tau^{\prime} e^{-\frac{1}{4} \frac{t^{2}}{\left(\tau-\tau^{\prime}\right)}} \frac{\mathfrak{j}_{1}\left(\tau^{\prime}\right)}{\left(\tau-\tau^{\prime}\right)^{3 / 2}}(. \mathrm{B}
$$

Lemmas $1,2,3$ lead to the following representation

$$
\begin{aligned}
e_{L}^{\tau \partial_{t}^{2}} \varphi(t) & =\frac{1}{2 \sqrt{\tau \pi}} \int_{0}^{\infty} d t^{\prime} e^{-\frac{\left(t-t^{\prime}\right)^{2}}{4 \tau}} \varphi\left(t^{\prime}\right) \\
& -\frac{1}{4 \sqrt{\pi}} \int_{0}^{\tau} d \tau^{\prime} e^{-\frac{1}{4} \frac{t^{2}}{\left(\tau-\tau^{\prime}\right)}} \frac{\phi\left(\sqrt{\tau^{\prime}}\right)-\phi\left(-\sqrt{\tau^{\prime}}\right)}{\sqrt{\tau^{\prime}} \sqrt{\tau-\tau^{\prime}}} \\
& +\frac{t}{8 \sqrt{\pi}} \int_{0}^{\tau} d \tau^{\prime} e^{-\frac{1}{4} \frac{t^{2}}{\left(\tau-\tau^{\prime}\right)}} \frac{\phi\left(\sqrt{\tau^{\prime}}\right)+\phi\left(-\sqrt{\tau^{\prime}}\right)}{\left(\tau-\tau^{\prime}\right)^{3 / 2}} .
\end{aligned}
$$


Proof of Proposition 1. We use the fact, that the inverse Laplace transform gives 0 for $t<0$

$$
\begin{aligned}
0 & =\frac{1}{2 \sqrt{\tau \pi}} \int_{0}^{\infty} d t^{\prime} e^{-\frac{\left(t+t^{\prime}\right)^{2}}{4 \tau}} \varphi\left(t^{\prime}\right) \\
& -\frac{1}{4 \sqrt{\pi}} \int_{0}^{\tau} d \tau^{\prime} e^{-\frac{1}{4} \frac{t^{2}}{\left(\tau-\tau^{\prime}\right)}} \frac{\phi\left(\sqrt{\tau^{\prime}}\right)-\phi\left(-\sqrt{\tau^{\prime}}\right)}{\sqrt{\tau^{\prime}} \sqrt{\tau-\tau^{\prime}}} \\
& -\frac{t}{8 \sqrt{\pi}} \int_{0}^{\tau} d \tau^{\prime} e^{-\frac{1}{4} \frac{t^{2}}{\left(\tau-\tau^{\prime}\right)}} \frac{\phi\left(\sqrt{\tau^{\prime}}\right)+\phi\left(-\sqrt{\tau^{\prime}}\right)}{\left(\tau-\tau^{\prime}\right)^{3 / 2}} .
\end{aligned}
$$

Therefore, subtracting (B.21) from $(\mathbb{B . 2 0})$ we get

$$
\begin{aligned}
\Psi_{N}(t, \tau)=e^{\tau \partial_{t}^{2}} \varphi(t) & =\frac{1}{2 \sqrt{\tau \pi}} \int_{0}^{\infty} d t^{\prime} e^{-\frac{\left(t-t^{\prime}\right)^{2}}{4 \tau}} \varphi\left(t^{\prime}\right)-\frac{1}{2 \sqrt{\tau \pi}} \int_{0}^{\infty} d t^{\prime} e^{-\frac{\left(t+t^{\prime}\right)^{2}}{4 \tau}} \varphi\left(\mathbf{B B}^{\prime} . \mathbf{2} 4\right) \\
& +\frac{t}{4 \sqrt{\pi}} \int_{0}^{\tau} d \tau^{\prime} e^{-\frac{1}{4} \frac{t^{2}}{\left(\tau-\tau^{\prime}\right)}} \frac{\phi\left(\sqrt{\tau^{\prime}}\right)+\phi\left(-\sqrt{\tau^{\prime}}\right)}{\left(\tau-\tau^{\prime}\right)^{3 / 2}} .
\end{aligned}
$$

that completes the proof of Proposition 1.

Lemma 5. The action of operator $e_{L}^{\tau \partial_{t}^{2}}$ on the constant is the same constant,

$$
e_{L}^{\tau \partial_{t}^{2}} 1=1
$$

Proof. Taking into account that

$$
\begin{aligned}
& \frac{1}{2 \sqrt{\tau \pi}} \int_{0}^{\infty} d t^{\prime}\left[e^{-\frac{\left(t-t^{\prime}\right)^{2}}{4 \tau}}-e^{\left.-\frac{\left(t+t^{\prime}\right)^{2}}{4 \tau}\right]}=\operatorname{erf}\left(\frac{1}{2} \frac{t}{\sqrt{\tau}}\right),\right. \\
& \frac{t}{2 \sqrt{\pi}} \int_{0}^{\tau} d \tau^{\prime} e^{-\frac{1}{4} \frac{t^{2}}{\left(\tau-\tau^{\prime}\right)}} \frac{1}{\left(\tau-\tau^{\prime}\right)^{3 / 2}}=-\operatorname{erf}\left(\frac{1}{2} \frac{t}{\sqrt{\tau}}\right)+1
\end{aligned}
$$

we get

$$
e_{L}^{\tau \partial_{t}^{2}} 1=\operatorname{erf}\left(\frac{1}{2} \frac{t}{\sqrt{\tau}}\right)-\operatorname{erf}\left(\frac{1}{2} \frac{t}{\sqrt{\tau}}\right)+1=1
$$

Here $\operatorname{erf}(t)$ is the error function,

$$
\operatorname{erf}(t)=\frac{2}{\sqrt{\pi}} \int_{0}^{t} e^{-x^{2}} d x .
$$

Note that $\operatorname{erf}(-t)=-\operatorname{erf}(t)$. 


\section{References}

[1] A. Linde, Inflation and Quantum cosmology, Academic Press, Boston, 1990.

[2] V.F. Mukhanov, Physical Foundations of Cosmology, Cambridge University Press, 2005.

[3] S. Weinberg, Gravity and Cosmology, Oxford Univ.Press, 2008.

[4] D.S. Gorbunov and V.A. Rubakov, Introduction to the Theory of the Early Universe, URSS, Moscow, 2009 (in Russian).

[5] S. Hawking, J. Ellis, The Large Scale Structure of Spacetime, Cambridge University Press, 1973.

[6] I.Ya. Aref'eva, Nonlocal String Tachyon as a Model for Cosmological Dark Energy, 2006 AIP Conf. Proc. 826, pp. 301-311, astro-ph/0410443

[7] I.Ya. Aref'eva, A.S. Koshelev, S.Yu. Vernov, Theor.Math.Phys. 148 (2006) 895, astro-ph/0412619.

[8] I.Ya. Aref'eva and L.V. Joukovskaya, 2005 Time lumps in nonlocal stringy models and cosmological applications, JHEP 0510, 087, [arXiv:hep-th/0504200].

[9] I.Ya. Aref'eva, A.S. Koshelev, S.Yu. Vernov, Stringy Dark Energy Model with Cold Dark Matte, Phys.Lett. B628 (2005) 1, astro-ph/0505605.

[10] I.Ya. Aref'eva, A.S. Koshelev, S.Yu. Vernov, Crossing of the $w=-1$ Barrier by D3-brane Dark Energy Model, Phys.Rev.D72:064017, 2005, astro-ph/0507067.

[11] G. Calcagni, Cosmological tachyon from cubic string field theory, JHEP 0605, 012 (2006), (hep-th/0512259)

[12] I.Ya. Aref'eva, A.S. Koshelev, Cosmic acceleration and crossing of $w=-1$ barrier in non-local Cubic Superstring Field Theory model, JHEP 0702:041, 2007, arXiv:hep-th/0605085

[13] I.Ya. Aref'eva and I.V. Volovich, On the null energy condition and cosmology, Theor. Math. Phys. 155, 503 (2008), arXiv:hep-th/0612098.

[14] N. Barnaby, T. Biswas, and J.M. Cline, p-adic Inflation, JHEP 0704 (2007) 056, (hep-th/0612230)

[15] A.S.Koshelev, Non-local SFT Tachyon and Cosmology, JHEP 0704029 (hep-th/0701103)

[16] Aref'eva I.Ya., Joukovskaya L.V. and Vernov S.Yu. 2007, Bouncing and accelerating solutions in nonlocal stringy models, JHEP 0707, 087 (hep-th/0701184)

[17] I.Ya. Aref'eva and I.V. Volovich, Quantization of the Riemann zeta-function and cosmology, Int. J. Geom. Meth. Mod. Phys. 4, 881 (2007) arXiv:hep-th/0701284. 
[18] J.E. Lidsey, Stretching the Inflaton Potential with Kinetic Energy, Phys. Rev. D 76 (2007) 043511, hep-th/0703007

[19] N. Barnaby and J.M. Cline, Large Nongaussianity from Nonlocal Inflation, JCAP 0707 (2007) 017, (arXiv:0704.3426)

[20] G. Calcagni, M. Montobbio and G. Nardelli, Route to nonlocal cosmology, Phys. Rev. D 76, 126001 (2007) arXiv:0705.3043

[21] L. Joukovskaya, Dynamics in Nonlocal Cosmological Models Derived from String Field Theory, Phys. Rev. D 76, 105007 (2007), (arXiv:0707.1545)

[22] N. Barnaby and N. Kamran, Dynamics with Infinitely Many Derivatives: The Initial Value Problem, JHEP 0802 (2008) 008 (arXiv:0709.3968)

[23] L. Joukovskaya, Rolling Tachyon in Nonlocal Cosmology, AIP Conf. Proc. 957 (2007) 325, arXiv:0710.0404

[24] I.Ya. Aref'eva, Stringy model of cosmological dark energy, 2007 AIP Conf.Proc. 957, 297-300, arXiv:0710.3017

[25] Aref'eva I.Ya., Joukovskaya L.V. and Vernov S.Yu. 2008 Dynamics in nonlocal linear models in the Friedmann-Robertson-Walker metric, J. Phys. A: Math. Theor. 41, 304003 (arXiv:0711.1364)

[26] G. Calcagni, G. Nardelli, 2008, Tachyon solutions in boundary and cubic string field theory, Phys. Rev. D 78, 126010, (arXiv:0708.0366)

[27] G. Calcagni, M. Montobbio, and G. Nardelli, Localization of nonlocal theories, Phys. Lett. B 662 (2008) 285-289, (arXiv:0712.2237)

[28] N. Barnaby and J. M. Cline, "Predictions for Nongaussianity from Nonlocal Inflation," JCAP 0806, 030 (2008) [arXiv:0802.3218].

[29] J. E. Lidsey, Non-local inflation around a local maximum, Int.J.Mod.Phys. D17:577-582,2008

[30] G. Calcagni, G. Nardelli, 2008, Nonlocal instantons and solitons in string models, Phys. Lett. B 669, 102-112, arXiv:0802.4395

[31] I. Ya. Aref'eva and A. S. Koshelev, JHEP 0809 (2008) 068 [arXiv:0804.3570 [hep-th]].

[32] Mulryne D.J. and Nunes N.J. 2008, Diffusing nonlocal infation: Solving the feld equations as an initial value problem, Phys. Rev. D 78063519 (arXiv:0805.0449)

[33] Joukovskaya L.V. 2009, Dynamics with Infinitely Many Time Derivatives in Friedmann-Robertson-Walker Background and Rolling Tachyon, JHEP 0902045 (arXiv:0807.2065) 
[34] N. Barnaby and N. Kamran, Dynamics with Infinitely Many Derivatives: Variable Coefficient Equations, JHEP 0812 (2008) 022 (arXiv:0809.4513)

[35] D.J. Mulryne and N.J. Nunes, Non-linear non-local Cosmology, AIP Conf. Proc. 1115 (2009) 329-334, (arXiv:0810.5471)

[36] N. Barnaby, D.J. Mulryne, N.J. Nunes and P. Robinson, 2009, Dynamics and Stability of Light-Like Tachyon Condensation, JHEP 0903, 018 (arXiv:0811.0608)

[37] N. Barnaby, Nonlocal Inflation, Can. J. Phys. 87 (2009) 189-194, (arXiv:0811.0814)

[38] Koshelev A.S. and Vernov S.Yu. Cosmological perturbations in SFT inspired nonlocal scalar field models, arXiv:0903.5176

[39] G. Calcagni, G. Nardelli, Kinks of open superstring field theory, Nucl. Phys. B 823 (2009) 234-253, arXiv:0904.3744

[40] G. Calcagni and G. Nardelli, 2010, Cosmological rolling solutions of nonlocal theories, Int. J. Mod. Phys. D 19, 329 (arXiv:0904.4245)

[41] G. Calcagni, G. Nardelli, 2010, String theory as a diffusing system, JHEP 1002, 093, arXiv:0910.2160

[42] S.Yu. Vernov, Localization of nonlocal cosmological models with quadratic potentials in the case of double roots, Class. Quant. Grav. 27 (2010) 035006, (arXiv:0907.0468)

[43] G. Calcagni, G. Nardelli, Non-local gravity and the diffusion equation, arXiv:1004.5144

[44] S.Yu. Vernov, Localization of the SFT inspired Nonlocal Linear Models and Exact Solutions, arXiv:1005.0372

[45] Vernov S.Yu., Exact Solutions for Nonlocal Nonlinear Field Equations in Cosmology, arXiv:1005.5007

[46] A.S. Koshelev and S.Yu. Vernov, Analysis of scalar perturbations in cosmological models with a non-local scalar field, arXiv:1009.0746

[47] F. Galli, A.S. Koshelev, Multi-scalar field cosmology from SFT: an exactly solvable approximation, Theor. Math. Phys. (2010) 164, 1169-1175 [Teor. Mat. Fiz. 164 (2010) 401-409] (arXiv:1010.1773)

[48] F. Galli and A. S. Koshelev, "Perturbative stability of SFT-based cosmological models," arXiv:1011.5672 [hep-th].

[49] I.Ya. Aref'eva, "Puzzles with Tachyon in SSFT and Cosmological Applications", Prog.Theor.Phys.Suppl.188:29-40,2011, arXiv:1101.5338 [hep-th]. 
[50] K. Ohmori, A Review on Tachyon Condensation in Open String Field Theories, hep-th/0102085;

I.Ya. Aref'eva, D.M. Belov, A.A. Giryavets, A.S. Koshelev, P.B. Medvedev, Noncommutative Field Theories and (Super)String Field Theories, hep-th/0111208.

W.Taylor and B.Zwiebach, D-Branes, Tachyons, and String Field Theory, hep-th/0311017;

W.Taylor, String field theory, hep-th/0605202

[51] I.V. Volovich, p-Adic String, Class.Quant.Grav. 4 (1987) L83.

L. Brekke, P.G.O. Freund, M. Olson, E. Witten, Nonarchimedean String Dynamics, Nucl. Phys. B302 (1988) 365.

P.H. Frampton, Ya. Okada, Effective Scalar Field Theory of P-Adic String, Phys. Rev. D37 (1988) 3077-3079.

[52] V.S. Vladimirov, I.V. Volovich, E.I. Zelenov, p-Adic Analysis and Mathematical Physics, World Sci., Singapore, 1994.

[53] B. Dragovich, A. Yu. Khrennikov, S. V. Kozyrev and I. V. Volovich, "On p-adic mathematical physics", p-Adic Numbers, Ultrametric Analysis and Applications, 2009, Vol. 1, No. 1, pp. 1-17; arXiv: 0904.4205.

[54] A.A. Starobinsky, Phys.Lett., B117 (1982) 175.

[55] I. Ya. Aref'eva, P. Frampton, B. Dragovich and I. V. Volovich. Wave function of the Universe and p-adic gravity, Inter.Mod.Phys., A 6 (1991) 4341-4358.

[56] V.S. Vladimirov, Ya.I. Volovich, Theor.Math.Phys. 138 (2004) 297-309, arXiv:math-ph/0306018.

[57] V.S. Vladimirov, The equation of the p-adic open string for the scalar tachyon field, Izvestiya: Mathematics, 69:3 (2005) 487-512, math-ph/0507018.

[58] D.V. Prokhorenko, On Some Nonlinear Integral Equation in the (Super)String Theory, math-ph/0611068

[59] L. Joukovskaya, Iterative method for solving nonlinear integral equations describing rolling solutions in string theory, Theor.Math.Phys.146 (2006), 335-342

(arXiv:0708.0642)

[60] V.S. Vladimirov, On the equations for p-adic closed and open strings, p-Adic Numbers, Ultrametric Analysis and Applications, 1(2009) 79-87.

[61] P. Gorka, H. Prado, E.G. Reyes, JMP, 51 (2010) 103512

[62] Davis H.T. 1936 The Theory of Linear Operators from the Standpoint of Differential Equations of Infinite Order, the Principia Press, Bloomington, Indiana, 1936 
[63] Carmichael R.D. 1936 Linear differential equations of infinite order, Bull. Amer. Math. Soc. 42 193-218

[64] Carleson L. 1953 On infinite differential equations with constant coefficients. I, Math. Scand. 1 31-38

[65] L. Hormander, The analysis of linear partial differential operators, Vol I: Pseudo-differential operators, 1983; Vol II: Differential operators with constant coefficients, 1983; Vol.III: Pseudo-differential operators, 1985; Vol.IV: Fourier integral operators, 1985

[66] Kh.A. Khachatryan, Solubility of a class of the second-order integro-differential equations with monotone non-linearity on a semi-axis, Izvestiya: Mathematics (2010), 74(5):1069-1082.

[67] A. Pais and G.E. Uhlenbeck, On Field theories with nonlocalized action, Phys. Rev. 79, 145 (1950).

[68] N. Moeller and B. Zwiebach, "Dynamics with infinitely many time derivatives and rolling tachyons," JHEP 0210, 034 (2002) [hep-th/0207107].

[69] Ya. I. Volovich, Numerical study of nonlinear equations with infinite number of derivatives, J. Phys. A36 (2003) 8685, math-ph/0301028

[70] I.Ya. Arefeva, L.V. Joukovskaya, A.S. Koshelev, JHEP 0309 (2003) 012. Time Evolution in Superstring Field Theory on non-BPS brane. I. Rolling Tachyon and EnergyMomentum Conservation, JHEP (2003) 0309 012, hep-th/0301137 .

[71] V.A. Fock, The eigen-time in classical and quantum mechanics, Izvestiya Akad. Nauk USSR, 1937, N 4-5, . 551-568. (in German) Phys. Zs. Sowjet., V. 12, N 4, (1937) 404-425.

[72] R.P. Feynman, Mathematical Formulation of the Quantum Theory of Electromagnetic Interaction, Phys. Rev. 80 (1950) 440-457

[73] D.A. Eliezer and R. P. Woodard, "The Problem of Nonlocality in String Theory," Nucl. Phys. B 325, 389 (1989).

[74] V.S. Vladimirov, Equations of Mathematical Physics, Moscow, Nauka, 1971

[75] M.E. Evgrafov, "Analitical functions, Nauka, Moscow, 1965.

[76] N.N. Bogolyubov, Y. A. Mitropolski, Asymptotic Methods in the Theory of Nonlinear Oscillations. Gordon and Breach, New York, 1961.

[77] A. A. Starobinsky, Phys. Lett. B 91, 99 (1980);

A. H. Guth, Phys. Rev. D 23, 347 (1981);

A. D. Linde, Phys. Lett. B 108, 389 (1982);

A. Albrecht and P. J. Steinhardt, Phys. Rev. Lett. 48, 1220 (1982) 
[78] A. D. Linde, Chaotic Inflation, Phys. Lett. B 129, 177 (1983).

[79] A. D. Linde, Initial Conditions For Inflation, Phys. Lett. B 162 (1985) 281.

[80] R.P. Woodard, J.Phys.Conf.Ser. 68:012032,2007; gr-qc/0608037.

[81] I.V. Volovich, Found Phys (2011) 41: 516528; arXiv:0907.2445.

[82] L. Accardi, Yu.G. Lu, I. Volovich, Quantum Theory and Its Stochastic Limit, Springer, 2002.

[83] L. E. Reichl, The Transition to Ghaos, Springer, 2004 